\title{
Bringing home the bacon? The next step in cardiac sodium channelopathies
}

\author{
Arthur A.M. Wilde ${ }^{1,2}$ and Pieter G. Postema ${ }^{1}$ \\ 'Department of Cardiology, Heart Center, Academic Medical Center, Amsterdam, The Netherlands. 2Princess Al-Jawhara Al-Brahim Centre of Excellence in Research of Hereditary Disorders, Jeddah, \\ Kingdom of Saudi Arabia.
}

\begin{abstract}
Mutations in SCN5A, which encodes the $\alpha$ subunit of the major cardiac sodium channel $\mathrm{Na}_{\mathrm{v}} 1.5$, are associated with multiple cardiac arrhythmias, including Brugada syndrome. It is not clear why mutations in SCN5A result in such a variety of cardiac phenotypes, and introduction of analogous Scn5a mutations into small-animal models has not recapitulated alterations in cardiac physiology associated with human disease. In this issue of the $\mathrm{JCl}$, Park and colleagues present a pig model of cardiac sodium channelopathy that was generated by introducing a human Brugada syndrome-associated SCN5A allele. This large-animal model exhibits many phenotypes seen in patients with SCN5A loss-of-function mutations and has the potential to provide important insight into sodium channelopathies.
\end{abstract}

\section{Cardiac sodium channel dysfunction}

The cardiac sodium channel is critical for heart function and richly embedded within cardiomyocyte membranes. Cardiac sodium channel dysfunction alters cardiac excitability, jeopardizing the safety of conduction and repolarization, which may result in malignant brady- or tachyarrhythmias and possibly sudden death. Pathologic alterations in cardiac sodium channel function are caused by mutations in genes encoding proteins involved in the construction of the channel or of other components of the macromolecular complex. The clinical manifestations of these mutations are herein referred to as cardiac sodium channelopathies (1). The majority of these inheritable arrhythmia syndromes are associated with a deviant function of the cardiac sodium channel due to mutations in SCN5A, which encodes the $\alpha$ subunit of the major cardiac sodium channel $\mathrm{Na}_{\mathrm{v}} 1.5(2-4)$. Since the identification of channelopathy-associated SCN5A muta- tions, the understanding of these syndromes has evolved enormously (1). Still, it is not clear why some loss-of-function mutations give rise to Brugada syndrome $(\mathrm{BrS})$, which is characterized by specific ECG changes and is a major risk factor for sudden cardiac death, and others do not. Moreover, the outcome of fully characterized $S C N 5 A$ mutations is not always predictable (5).

\section{Phenotypes of cardiac sodium channelopathies}

Loss-of-function SCN5A mutations are associated with several different phenotypes, including progressive cardiac conduction defects (PCCD), also known as Lenegre-Lev disease (4), and BrS (3, 6). PCCD is characterized by progressive conduction slowing in the His-Purkinje system, giving rise to $\mathrm{PR}$ interval lengthening, QRS widening, and ultimately complete atrioventricular (AV) block with syncope and/or sudden death (4). BrS, characterized by a signature covedtype ST segment in the right precordial

Related Article: p. 403

Conflict of interest: The authors have declared that no conflict of interest exists.

Reference information: / Clin Invest. 2015;125(1):99-101. doi:10.1172/JCI80014.

ECG leads, is strongly associated with delayed conduction parameters (in all cardiac compartments) and malignant arrhythmias. In contrast to Lenegre-Lev disease, the underlying pathophysiologic mechanisms of the ST segment elevation in BrS are heavily debated (7). The current understanding of these conditions has matured from many preclinical studies meticulously analyzing SCN5A mutations in cells and small-animal models such as mice; however, there remains a rather large gap between these preclinical experiments and human disease, as human whole-heart physiology has not been be truly recapitulated in these model systems.

\section{Bringing home the bacon}

In this issue, Park and colleagues have taken the next step in the preclinical study of cardiac sodium channelopathies and generated a large-animal model of a loss-offunction sodium channelopathy (8) that has allowed an impressive array of in vivo and ex vivo investigations. Specifically, the authors engineered a Yucatan mini pig population that carries an early truncation mutation in SCN5A (E555X). Notably, due to housing limitations and cost, these animals were studied well before reaching the age of 2 years, which is considered an adult age (albeit relatively young). Yucatan pigs have a life span of 10-15 years, reach sexual maturity in 4-6 months, and weigh approximately $30 \mathrm{~kg}$ at 6 months and $45-50 \mathrm{~kg}$ at 1 year.

These $S C N 5 A^{E 558 X /+}$ mutant animals exhibited conduction abnormalities at all cardiac levels (atrium, AV node, and specialized conduction system and ventricle) in the absence of gross structural cardiac defects; however, no elevation of the right precordial ST segment was observed. Notably, only female animals were used for this part of the analysis (see below). In male animals, in vivo endocardial and epicardial mapping revealed an 
absence of low-voltage signals, which are indicative of fibrosis, but EGM duration was slightly longer in the right ventricular outflow tract (RVOT) area. Functional and pathoanatomical in vivo analysis did not reveal any abnormalities. No sudden cardiac death was observed in any of the animals; however, in the Langendorff setup, isolated perfused hearts from $S C N 5 A^{E 558 X /+}$ animals had an increased propensity for pacing-induced and spontaneous ventricular fibrillation compared with hearts from control animals. These arrhythmias were initiated by short-coupled ventricular premature beats and, interestingly enough, were very sensitive to temperature. In isolated mutant hearts, conduction velocity was significantly reduced (throughout the right ventricle), whereas action potential durations were not altered (8).

Inhibition of the $\mathrm{Na}_{\mathrm{v}} 1.5$ with flecainide resulted in further conduction slowing, which was more pronounced in adult animals in this cohort $(15 \pm 2$ months), but still did not result in BrStype changes. It should be noted that only female animals were studied in these experiments and that the conduction delay induced by flecainide provocation was progressive with age. In the expression analyses, it was also documented that the mutant cells exhibited diminished levels of the sodium channel protein and decreased current density, although the localization of the macromolecular sodium channel complex in the intercalated discs was unaltered in SCN5A ${ }^{E 558 X /+}$ mutant animals (8).

\section{Discussion}

The phenotype of the $S C N 5 A^{5558 X /+}$ pigs is clearly indicative of loss of sodium channel function (i.e., PCCD), but these animals do not present with a BrS-type ECG. Importantly, the orthologous human mutation selected by Park and colleagues, E558X, was initially identified in a young child who presented with fever, atrial flutter, nonsustained polymorphic ventricular tachycardia, and a BrS type 1-pattern ECG (8). The baseline ECG of this child was not indicative of typical loss-of-function sodium channelopathies, although the conduction intervals observed in the patient were at the upper limit of the normal range for a 10-year-old.
After flecainide administration, the ECG of this patient was more or less consistent with BrS type 1 (8).

For numerous reasons, it should not come as a complete surprise that this genetically modified animal model, especially evaluated at a young age, was characterized by progressive conduction disease rather than displaying a BrS-associated ECG pattern. SCN5A mutation type, age of onset, and gender all potentially play a role in the overt presentation of this disease. The presence of one truncated $S C N 5 A^{E 558 X}$ allele in the pig model will lead to haploinsufficiency, with an estimated $50 \%$ of the sodium channels remaining functional. Compared with those with missense mutations, patients with truncating $S C N 5 A$ mutations present with more pronounced conduction disease (9). Moreover, exclusive conduction disease, without identifiable BrS, was previously described in several families with SCN5A mutations leading to haploinsuffiency $(4,10-12)$. It should be noted, however, that not all of these were truncation mutations, although the patients presented with haploinsuffiency behavior. It is well known that BrS is very rare in children (13), and even with the presence of a loss-of-function SCN5A mutation, the vast majority of children present with conduction disease rather than a BrSassociated ECG pattern at baseline (14). Gender also contributes to the presentation of BrS: evaluation of a large family with an $S C N 5 A$ mutation that resulted in nonfunctional sodium channels showed BrS-associated signs only in males that carried the mutant allele (4 of 7), whereas all 6 female $S C N 5 A$ mutant carriers presented with conduction disease only (15). Park and colleagues analyzed their ECG data only in female animals, although females within the patient study were prone to conduction disease only, not BrS (8). In the male pigs studied, the results were apparently similar to those in females (D. Park and G. Fishman, unpublished observation). The authors suggest that the lack of observed transient outward current in the mutant pig heart may be causally related to the absence of the BrS-associated ECG in pigs. Recent studies strongly indicate that BrS ECG presentations are linked to safety of conduction, which is indeed dependent on transient outward current amplitude (16) and/or on sodium current, as shown in the present study (8).

The age-dependent penetrance of the BrS phenotype in patients is intriguing. Most likely, there is a combined role for testosterone levels (17) and the presence of progressive fibrosis development. Agerelated fibrosis has indeed been shown in a haploinsufficient $S c n 5 a$ mouse model $\left(S c n 5 a^{+/-}\right.$mice), with resultant progressive impairment of atrial and ventricular conduction (18). It is not known whether the individuals in published pediatric cases that are described as merely having conduction disease (14) will go on to develop a BrS-type ECG later in life. Similarly, it remains to be seen whether the mutant Yucatan pigs developed by Park et al. will develop a BrS pattern when they mature. It is quite likely that there could be an age-dependent mechanism that promotes BrS.

\section{Conclusions}

Park and colleagues have indeed taken a long-awaited step in the preclinical study of sodium channelopathies. This Yucatan mini pig loss-of-function sodium channelopathy model (8) will allow both classic preclinical and clinical investigations to be meticulously performed and provides a new tool to help address the vast number of uncertainties in these diseases. It will be in the best interest of the field for this model to be further studied. Future detailed analysis that includes older animals will undoubtedly be extremely interesting, and the comparison of female with male animals throughout the whole age range will be equally important.

\section{Acknowledgments}

The authors acknowledge support from the Netherlands CardioVascular Research Initiative: the Dutch Heart Foundation, Dutch Federation of University Medical Centres, the Netherlands Organisation for Health Research and Development, and the Royal Netherlands Academy of Sciences.

Address correspondence to: Arthur A.M. Wilde, Department of Cardiology, Academic Medical Centre - University of Amsterdam, P.O. Box 22660, 1100 DD Amsterdam, The Netherlands. Phone: 020.566.29.04; E-mail: a.a.wilde@amc.nl. 
1. Schwartz PJ, Ackerman MJ, George AL Jr, Wilde AAM. Impact of genetics on the clinical management of channelopathies. J Am Coll Cardiol. 2013;62(3):169-180.

2. Wang $Q$, et al. SCN5A mutations associated with an inherited cardiac arrhythmia, long QT syndrome. Cell. 1995;80(5):805-811.

3. Chen $\mathrm{Q}$, et al. Genetic basis and molecular mechanism for idiopathic ventricular fibrillation. Nature. 1998;392(6673):293-296.

4. Schott JJ, et al. Cardiac conduction defects associate with mutations in SCN5A. Nat Genet. 1999;23(1):20-21.

5. Postema PG, Mosterd A, Hofman N, Alders M, Wilde AA. Sodium channelopathies: do we really understand what's going on? J Cardiovasc Electrophysiol. 2011;22(5):590-593.

6. Rook MB, et al. Human SCN5A gene mutations alter cardiac sodium channel kinetics and are associated with the Brugada syndrome. Cardiovasc Res. 1999;44(3):507-517.

7. Wilde AA, et al. The pathophysiological mechanism underlying Brugada syndrome: depolariza- tion versus repolarization. JMol Cell Cardiol. 2010;49(4):543-553.

8. Park DS, et al. Genetically engineered SCN5A mutant pig hearts exhibit conduction defects and arrhythmias. JClin Invest. 2015;125(1):403-412.

9. Meregalli PG, et al. Type of SCN5A mutation determines clinical severity and degree of conduction slowing in loss-of-function sodium channelopathies. Heart Rhythm. 2009;6(3):341-348.

10. Tan HL, et al. A sodium-channel mutation causes isolated cardiac conduction disease. Nature. 2001;409(6823):1043-1047.

11. Probst V, et al. Haploinsufficiency in combination with aging causes SCN5A-linked hereditary Lenegre disease. J Am Coll Cardiol. 2003;41(4):643-652.

12. Zumhagen S, et al. A heterozygous deletion mutation in the cardiac sodium channel gene SCN5A with loss- and gain-of-function characteristics manifests as isolated conduction disease, without signs of Brugada or long QT syndrome. PLoS One. 2013;8(6):e67963.
13. Probst V, et al. Clinical aspects and prognosis of Brugada syndrome in children. Circulation. 2007;115(15):2042-2048.

14. Chockalingam P, et al. The diagnostic and therapeutic aspects of loss-of-function cardiac sodium channelopathies in children. Heart Rhythm. 2012;9(12):1986-1992.

15. Kyndt F, et al. Novel SCN5A mutation leading either to isolated cardiac conduction defect or Brugada syndrome in a large French family. Circulation. 2001;104(25):3081-3086.

16. Hoogendijk MG, Potse M, Vinet A, Bakker JM, Coronel R. ST segment elevation by current-toload mismatch: an experimental and computational study. Heart Rhythm. 2011;8(1):111-118.

17. Shimizu W, et al. Sex hormone and gender difference - role of testosterone on male predominance in Brugada syndrome. JCardiovasc. 2007;18(4):415-421.

18. Royer A, et al. Mouse model of SCN5A-linked hereditary Lenègre's disease: age-related conduction slowing and myocardial fibrosis. Circulation. 2005;111(14):1738-1746. 